\title{
Discrimination of Chiral Guests by Chiral Channels: Variable Temperature Studies by SXRD and Solid State 13C NMR of the Deoxycholic Acid Complexes of Camphorquinone and Endo-3-Bromocamphor
}

\begin{abstract}
3a,12a-Dihydroxy-5b-cholan-24-oic acid (deoxycholic acid DCA) is able to discriminate between the $\mathrm{R}$ - and S-enantiomers of camphorquinone and endo-(1)-3-bromocamphor and select only the S-enantiomers from a racemic mixture. DCA forms novel well ordered 1:1 adducts with (1S)-(1)-camphorquinone and (1S)-endo-(-)-3-bromocamphor, both of which have been characterized by single crystal X-ray diffraction SXRD). When DCA is cocrystallized with (RS)-camphorquinone and (RS)-endo-3-bromocamphor,1:1 adducts of the S-enantiomers are produced together with crystals of the free racemic guest. In contrast, in the absence of (1S)-(1)-camphorquinone, DCA forms a 2:1 adduct with (1R)-(2)camphorquinone. In this 2:1 adduct the guest is disordered at ambient temperature and undergoes a phase change in the region 160-130 K similar to that observed for the ferrocene adduct, but with only partial ordering of the guest. The SXRD structure of the low temperature form and the variable temperature 13C CP/MAS NMR are reported. Cocrystallizing DCA with (1R)-endo-(1)-3-bromocamphor gives the free guest and a glassy solid.
\end{abstract}

Keyword: molecular complexes, chiral discrimination, chiral selection 